DOI: 10.12957/demetra.2016.19641

\title{
Comercialização de lanches e bebidas em escolas públicas: análise de uma regulamentação estadual
}

\section{The sale of snacks and beverages in public schools: analysis of a state regulatory}

José Divino Lopes Filho

Larissa Loures Mendes²

' Universidade Federal de Minas Gerais, Escola de Enfermagem, Departamento de Nutrição. Belo Horizonte-MG, Brasil.

${ }^{2}$ Universidade Federal de Juiz de Fora, Instituto de Ciências Biológicas, Departamento de Nutrição. Juiz de Fora-MG, Brasil.

Correspondência / Correspondence José Divino Lopes Filho

Universidade Federal de Minas Gerais, Escola de Enfermagem/Departamento de Nutrição. Av. Alfredo Balena, 190, sala 316, CEP 30130100, Belo Horizonte-MG, Brasil.

E-mail:divinolopes@gmail.com.

\section{Resumo}

O incentivo à alimentação adequada e saudável nas escolas é prática comum em vários países e tem sido cada vez mais regulamentado em municípios e estados brasileiros. Entretanto, as leis criadas parecem não garantir impedir que a qualidade de todos os alimentos disponíveis nessas instituições seja adequada. No Estado de Minas Gerais, a Lei no 18.372 , de 2009, regulamenta a venda de lanches e bebidas de baixo valor nutricional nas escolas estaduais. $\mathrm{O}$ objetivo foi verificar o cumprimento da regulamentação do comércio de lanches e bebidas em escolas públicas estaduais do município de Belo Horizonte. Foram avaliadas 156 escolas que correspondem a $87 \%$ do total. Para a coleta dos dados, foi utilizado questionário padronizado desenvolvido pela Coordenadora do Programa de Alimentação Escolar de Minas Gerais. Foram coletados dados sobre a infraestrutura das escolas e alimentos comercializados em seu interior. Foi elaborado um banco de dados a partir do qual se investigou o cumprimento da lei, por meio da comparação do que foi comercializado com as restrições previstas em lei. Verificou-se que em 78 escolas $(50,6 \%)$ havia comercialização de alimentos com excesso de gordura trans, saturada, sódio, açúcares e calorias. As frequências encontradas de venda de alimentos proibidos pela lei foram as seguintes: salgados assados, $82,4 \%$; bebidas artificiais, refrigerantes e sucos artificiais, $54,8 \%$; embutidos, $42,7 \%$; bacon, batata palha, sorvetes e molhos gordurosos, $34,2 \%$. A regulamentação da venda de alimentos nas escolas pode ser considerada etapa importante na promoção da alimentação saudável dos escolares, mas faz-se necessária a constante fiscalização para que haja o cumprimento da legislação. 
Palavras-chave: Alimentação escolar. Saúde escolar. Hábitos alimentares. Comercialização de Produtos.

\section{Abstract}

Encouraging an adequate and healthy food in schools is a common practice in many countries and has been increasingly regulated in the municipalities and states. However, the laws created do not seem to guarantee that the quality of all the food available in these institutions is appropriate. In the state of Minas Gerais, the Law No. 18,372 of 2009 regulates the sale of snacks and beverages of low nutritional value in state schools. The objective was to verify compliance with regulatory trade for snacks and drinks in public schools in the city of Belo Horizonte. A total of 156 schools which correspond to $87 \%$ of the total were evaluated. For data collection, a standardized questionnaire developed by the Coordinator of the School Feeding Program of Minas Gerais was used. The data of the infrastructure of schools and the food sold inside there were collected. A database was developed from which it could investigate the compliance with the law, comparing what was marketed with the restrictions established by law. It was verified that 78 schools $(50.6 \%)$ were selling foods with the excess of Trans fat, saturated fat, sodium, sugars and calories. The frequencies found of the selling of food prohibited by law were: salty baked goods, $82.4 \%$; artificial beverages, soft drinks and artificial juices, $54.8 \%$; embedded derivate, $42.7 \%$; bacon, straw potato, ice cream and greasy sauces, $34.2 \%$. The regulation of the food sales in schools can be considered an important step in promoting healthy eating at school, but the constant supervision is necessary for compliance with legislation.

Key words: School feeding. School health. Food habits. Products commerce.

\section{Introdução}

Ações de promoção da saúde nas escolas têm sido implementadas em vários países, como reconhecimento à posição estratégica desse ambiente na sociedade. ${ }^{1,2,3,4}$ Dentre as ações de promoção de saúde, o incentivo à alimentação adequada e saudável ocupa lugar de destaque, pois é nesse ambiente que crianças e adolescentes permanecem por expressivo período de tempo. Nesse sentido, é fundamental perceber a importância que os serviços de alimentação disponíveis no ambiente escolar devem assumir, principalmente no que se refere ao fornecimento ou comercialização de alimentos e refeições adequadas e saudáveis..$^{5,6}$ 
Contudo, estudos evidenciam que os alimentos disponíveis nas escolas, especialmente os ofertados em lanchonetes comerciais, são produtos normalmente com baixo teor nutricional e alto valor energético. Além disso, destaca-se que as preferências alimentares dos mais jovens nem sempre recaem sobre os alimentos considerados mais saudáveis. Nesse contexto, o ato alimentar pouco saudável contribui para o aparecimento e agravo das Doenças Crônicas Não Transmissíveis (DCNT), o que corrobora uma crescente prevalência de obesidade entre escolares., ${ }^{7,9}$

Dados da Pesquisa Nacional de Saúde do Escolar (PeNSE) realizada em 2012 mostraram que o consumo pelos estudantes de alimentos considerados não saudáveis, ricos em açúcares e gorduras, tem aumentado. O consumo de guloseimas (doces, balas, chocolates, chicletes, bombons ou pirulitos) em cinco dias ou mais na semana foi referido por 41,3\% dos escolares, em conjunto com o consumo de biscoitos salgados $(35,1 \%)$ e de refrigerantes $(33,2 \%)$. Estes dados reafirmam as conclusões observadas na PeNSE de 2009 acerca do padrão regular e elevado do consumo de alimentos não saudáveis por parcela significativa dos estudantes brasileiros. ${ }^{10}$

As informações da PeNSE, por refletirem práticas e legislações que orientam a disponibilidade e consumo de alimentos no ambiente escolar, são relevantes para o planejamento de intervenções de educação alimentar e nutricional (EAN). Conclusões de estudos diversos têm avalizado os efeitos positivos dessas ações envolvendo o ambiente escolar. ${ }^{11,12,13,14}$

Deve-se ressaltar que as experiências estaduais e municipais de regulamentação da comercialização de alimentos em escolas responde às diretrizes do Programa Nacional de Alimentação Escolar (PNAE) que, por sua vez, é um dos principais instrumentos de promoção do direito humano à alimentação adequada (DHAA). ${ }^{15,16,17}$

Ressalta-se ainda que a comercialização de alimentos de elevada densidade energética pelas cantinas representa um entrave para as ações de EAN. A presença desses estabelecimentos nas escolas pode interferir diretamente na adesão dos alunos às ações do PNAE, tendo em vista que as refeições distribuídas pelo programa são elaboradas com base em parâmetros nutricionais e recomendações para promoção de uma alimentação saudável. ${ }^{6}$

Diante desse contexto, o Estado de Minas Gerais, em 4 de setembro de 2009, aprovou e sancionou a Lei no 18.372, que regulamenta a venda de lanches e bebidas no âmbito das escolas do sistema estadual de ensino. Por meio da mesma

fica vedada, nos espaços das escolas estaduais, a comercialização de lanches e bebidas contendo os produtos ou preparações, industrializados ou não, que contenham altos teores de calorias, gordura saturada, gordura trans, açúcar livre, sal, teor alcoólico e baixo teor nutricional, tais como: frituras, salgados e doces com massa folhada, biscoitos, doces em geral, molhos calóricos, bebidas artificiais, salgadinhos e pipocas industrializadas, embutidos, sanduíches e pizza ricos em gorduras e calorias. ${ }^{18}$ 
A elaboração de portarias por gestores dos estados e municípios que regulamentam o funcionamento das cantinas escolares pode ser considerada um importante passo em direção à transformação da escola em um ambiente facilitador para escolhas alimentares adequadas. Aliada a esta regulamentação destaca-se a importância das ações de EAN que envolvam alunos e familiares para a consolidação de hábitos alimentares saudáveis. ${ }^{6}$

Sabendo da importância de uma alimentação adequada e saudável para o crescimento e desenvolvimento das crianças e adolescentes, o presente trabalho verificou se a promoção do DHAA está sendo efetivada em escolas da rede pública estadual da região metropolitana de Belo Horizonte, com base no cumprimento da Lei 18.372/2009, que restringe a comercialização de alimentos não saudáveis em lanchonetes e cantinas escolares $\mathrm{O}$ objetivo foi verificar o cumprimento da regulamentação do comércio de lanches e bebidas em escolas públicas estaduais da região metropolitana de Belo Horizonte.

\section{Métodos}

Trata-se de um estudo transversal, realizado em todas as escolas estaduais da Região Metropolitana de Belo Horizonte (RMBH) cadastradas no ano de 2012 na Secretaria Estadual de Educação de Minas Gerais, que correspondiam a um total de 156 escolas. Dessas, 78 escolas (50\%) comercializavam produtos alimentícios nas cantinas e todas foram selecionadas para compor a amostra do presente estudo. Os dados utilizados na pesquisa foram disponibilizados pela Diretoria de Suprimento Escolar da SEE-MG e coletados durante um ano uma vez em cada escola pelas nutricionistas da supervisão técnica do programa de alimentação escolar.

O estudo investigou quais são os tipos de lanches comercializados nessas escolas a partir de dados coletados pela Diretoria de Suprimento Escolar da Secretaria de Estado de Educação de Minas Gerais (SEE-MG).

Para a coleta dos dados, a SEE-MG utiliza um questionário que aborda questões referentes à infraestrutura da escola e à alimentação fornecida no ambiente escolar. Dentre as questões estão informações como: número de alunos matriculados, dados sobre a aquisição e controle de gêneros alimentícios, informações sobre os cardápios, controle de estoque, recursos humanos e comercialização de alimentos e bebidas.

No presente estudo, foram analisadas as questões referentes ao comércio de alimentos dentro do ambiente escolar, de modo a verificar se as escolas atendiam à Lei no 18.372, de 2009, que veda e limita o comércio de alguns tipos de alimentos e preparações. Os alimentos avaliados pela SEE-MG são alimentos processados e ultraprocessados, descritos no Quadro 1.

A análise descritiva dos dados foi realizada por meio da distribuição de frequência com o auxílio do software Excel 14.4.7 da Microsoft. 
Quadro 1. Categorias de alimentos processados e ultraprocessados comercializados nas escolas.

\begin{tabular}{|c|c|}
\hline CATEGORIAS & ALIMENTOS \\
\hline Frituras & $\begin{array}{l}\text { Batatas, biscoitos, bolinhos, coxinhas, } \\
\text { enroladinhos recheados, espetinhos, pastéis, } \\
\text { quibes e frituras em geral }\end{array}$ \\
\hline $\begin{array}{c}\text { Salgados Assados / Salgados com } \\
\text { massa folhada }\end{array}$ & Salgados e doces \\
\hline Biscoitos & $\begin{array}{l}\text { Biscoitos recheados, com cobertura, tipo wafer, } \\
\text { biscoitos salgados e outros com alto teor de } \\
\text { gorduras e calorias }\end{array}$ \\
\hline Doces & $\begin{array}{l}\text { Balas, pastilhas, pirulitos, chocolates e } \\
\text { bombons, suspiros, maria-mole, sorvetes de } \\
\text { massa, picolés de massa com cobertura, chupe- } \\
\text { chupe, algodão doce, gomas de mascar e } \\
\text { guloseimas em geral }\end{array}$ \\
\hline Molhos calóricos & $\begin{array}{l}\text { Catchup, maionese, mostarda, molhos à base de } \\
\text { maionese e outros com alto teor de gorduras e } \\
\text { calorias }\end{array}$ \\
\hline Bebidas artificiais & $\begin{array}{l}\text { Refrigerante comum, light e zero, refrescos } \\
\text { artificiais, bebidas alcoólicas, energéticos e } \\
\text { outras bebidas similares }\end{array}$ \\
\hline $\begin{array}{l}\text { Outros alimentos prontos para } \\
\text { consumo }\end{array}$ & $\begin{array}{l}\text { Salgadinhos industrializados, sanduíches e } \\
\text { pizzas que contenham ingredientes como } \\
\text { embutidos, bacon, batata palha, maionese e } \\
\text { molhos gordurosos e calóricos, ovos fritos, } \\
\text { queijos gordurosos e outros ingredientes e } \\
\text { embutidos ricos em gorduras e caloria }\end{array}$ \\
\hline Outros alimentos & $\begin{array}{l}\text { Alimentos que apresentam baixo valor } \\
\text { nutricional, mas que não foram anteriormente } \\
\text { descritos. }\end{array}$ \\
\hline
\end{tabular}




\section{Resultados e Discussão}

Das 78 escolas que comercializavam produtos alimentícios nas cantinas, em 90,9\% (n=70) o gerenciamento era realizado pela própria administração escolar. Quanto à comercialização dos alimentos processados e ultraprocessados proibidos pela Lei no 18.372/2009, verificou-se uma elevada frequência da disponibilidade para comercialização desses alimentos nas cantinas, destacando, entre eles, os salgados assados, que foram os mais disponíveis $(82,4 \%)$ seguidos das bebidas artificiais, refrigerantes e sucos artificiais (54,8\%). Embutidos como salsicha (42,7\%) estavam presentes em preparações, e outros produtos como bacon e a batata palha. Verificou-se ainda que molhos gordurosos (34,2\%) também eram disponibilizados para consumo nas cantinas.

Os alimentos que tiveram menor percentual de disponibilidade para comercialização nas cantinas - apesar de restritos pela legislação - foram os alimentos fritos (9,3\%), os sanduíches $(10,7 \%)$, os doces $(25,3 \%)$ e os salgadinhos industrializados $(17,3 \%)$ (Tabela 1$)$.

Tabela 1. Alimentos e bebidas comercializados pelas cantinas escolares da rede estadual da RMBH, 2012.

\begin{tabular}{cccccc}
\hline & \multicolumn{2}{c}{ Sim } & \multicolumn{2}{c}{ Não } & Total \\
\cline { 2 - 5 } Alimentos & $\mathrm{n}$ & $\%$ & $\mathrm{n}$ & $\%$ & \\
\hline Salgados Assados & 61 & 82,4 & 13 & 17,6 & 74 \\
Bebidas Artificiais & 40 & 54,8 & 33 & 45,2 & 73 \\
Embutidos & 32 & 42,7 & 43 & 57,3 & 75 \\
Outros Produtos* & 26 & 34,2 & 50 & 65,8 & 76 \\
Doces & 19 & 25,3 & 56 & 74,7 & 75 \\
Salgadinhos & 13 & 17,3 & 62 & 82,7 & 75 \\
Sanduíche & 8 & 10,7 & 67 & 89,3 & 75 \\
Salgadinhos & 13 & 17,3 & 62 & 82,7 & 75 \\
Industrializados & & & & & 75 \\
Alimentos Fritos & 7 & 9,3 & 68 & 90,7 & 75 \\
\hline
\end{tabular}

Nota:* Venda de Outros Produtos: Alimentos com ingredientes, como bacon, batata palha e molhos gordurosos. 
Diversos estudos apontam que expressiva parcela dos alunos das escolas públicas consome alimentos comercializados por essas lanchonetes. Ressalta-se que a presença de dois tipos de serviços de alimentação nas escolas, oferta de alimentação escolar e comercialização de alimentos pelas cantinas, possivelmente gera dificuldades para que os estudantes selecionem, de maneira mais adequada, os alimentos que devem integrar sua alimentação no período escolar. ${ }^{6}$

Foi possível identificar neste estudo a venda para as escolas de produtos proibidos pela legislação estadual vigente, como: bebidas artificiais, embutidos, bacon, batata palha e molhos gordurosos. Constatou-se que os salgados assados apresentaram o maior percentual de venda $(82,4 \%)$, constituindo-se uma modalidade de produtos liberados para a comercialização nas cantinas (com exceção das massas folhadas). Verifica-se que esses produtos ocupam espaço cada vez maior nas cantinas escolares, o que pode ser um ponto positivo devido à redução da disponibilização de frituras (disponíveis em apenas 9,3\% dos estabelecimentos escolares). Porém, é válido atentar-se a esse resultado, já que muitos salgados mesmo sendo assados podem representar um risco à saúde por conterem altos teores de gordura trans e saturada, como é o caso dos salgados folhados tipo de massa vedada pela legislação. Contudo, uma das limitações do presente estudo foi o não detalhamento dos ingredientes das preparações.

No que diz respeito às bebidas artificiais, como os refrigerantes comum, light e zero e sucos industrializados, verificou-se que esse tipo de produto teve o segundo maior percentual de vendas $(54,8 \%)$. Deve-se destacar que ambos os produtos (salgados assados e bebidas artificiais) foram os alimentos que apresentaram percentual de venda superior a 50\%. Estudo realizado por Gabriel et al. ${ }^{19} \mathrm{em}$ cantinas de escolas públicas e particulares de Florianópolis, também avaliou os alimentos e bebidas comercializados após a instituição de lei municipal que regulamenta o funcionamento das cantinas escolares no município. Essa lei proíbe a comercialização de bebidas alcoólicas; balas, pirulitos e gomas de mascar; refrigerantes e sucos artificiais; salgadinhos industrializados; salgados fritos e pipocas industrializadas. No estudo, verificou-se o alto percentual de venda de salgados assados $(98,2 \%)$ e sucos artificiais $(60,7 \%)$ e venda de embutidos e outros produtos à base de bacon, batata palha e molhos gordurosos, que também apresentaram frequência elevada, de $42,7 \%$ e $34,2 \%$, respectivamente.

Diante dos resultados do presente estudo, entende-se que a Lei n 18.372/2009 ainda não é aplicada em sua totalidade na RMBH. Ressalta-se que em algumas escolas alimentos processados e ultraprocessados encontram-se acessíveis aos alunos, o que implica descumprimento da lei. Além disso, a presença desses estabelecimentos nas escolas pode interferir diretamente na adesão dos alunos ao Programa Nacional de Alimentação Escolar (PNAE). 
É importante considerar que a escola deve ser vista como um espaço de promoção para uma alimentação adequada e saudável, sendo um local ideal para ações de EAN. ${ }^{19,20,21}$ Ressalta-se a importância de programas de EAN que envolvam alunos, familiares, gestores e educadores, para que não permitam a comercialização desses produtos proibidos por lei e também para adesão a essas estratégias saudáveis de alimentação no ambiente escolar.

De acordo com a própria Lei 18.372, a fiscalização para verificação do seu cumprimento deve ser realizada pelos serviços de Vigilância Sanitária dos municípios e do estado. Além disso, a resolução que regulamenta a aplicação desta lei atribui ao diretor ou coordenador da unidade de ensino a responsabilidade de também fiscalizar o seu cumprimento. Quanto à Vigilância Sanitária, incorporou a lei como referência para elaboração de protocolo de ações da área e em alguns municípios a atividade de fiscalização ocorre. A violação da lei pode representar, quando se tratar de cantina terceirizada, ruptura do contrato de uso do espaço e exploração do serviço. Quando se tratar de descumprimento realizado por servidor público, preveem-se penalidades administrativas inclusas no Estatuto do Servidor Público. Apesar desses instrumentos legais para aferição do cumprimento da lei, o presente estudo não identificou dados que revelem números de escolas e resultados de fiscalização das mesmas. ${ }^{22,23}$

\section{Conclusão}

A elaboração de portarias por gestores dos estados e municípios que regulamentam o funcionamento das cantinas pode ser considerada um importante passo em direção à transformação da escola em um ambiente facilitador para escolhas alimentares adequadas. Porém, faz-se necessária a constante fiscalização para que haja o cumprimento da legislação em todas as escolas, considerando que a legislação vigente atribui poder de fiscalização não apenas aos serviços de vigilância sanitária, mas também ao responsável pela unidade de ensino. A ausência de informações sobre as atividades de fiscalização deixa em aberto importante instrumento de controle social da Lei 18.372, resultando em desconhecimento do que é praticado nas escolas.

\section{Agradecimentos}

Agradecemos ao apoio de Valéria Monteiro, da Secretaria de Estado de Educação de Minas Gerais, e de Rafael Claro, do Departamento de Nutrição da Universidade Federal de Minas Gerais. 


\section{Referências}

1. Hurrelmann K, Leppin A, Nordlohne E. Promoting health in schools: the German example. Health Promot Internation 1995; 10(2):121-131.

2. Peru. Ministerio de Salud. Dirección General de Promoción de La Salud. Programa de Promoción de la Salud en las Instituciones Educativas: escuelas saludables. Lima: Dirección General de Promoción de La Salud; 2005.

3. St Leger L, Young IM. Creating the document 'promoting health in schools: from evidence to action'. Glob Health Promot 2009; 16(4):69-71.

4. Tanaka N, Miyoshi M. School lunch program for health promotion among children in Japan. Asia Pac J Clin Nutr. 2012; 21(1):155-158.

5. Pilant VB. Position of the American Dietetic Association: local support for nutrition integrity in schools. J Am Diet Assoc. 2006; 106(1):122-133.

6. Danelon MAS, Danelon MS, Silva MV. Serviços de alimentação destinados ao público escolar: análise da convivência do Programa de Alimentação Escolar e das cantinas. Segur Aliment Nutr. 2006; 13(1):85-94.

7. World Health Organization. Global health risks: mortality and burden of disease attributable to selected major risks. Geneva: WHO; 2009.

8. Li J, Hooker NH. Childhood obesity and schools: evidence from the national survey of children's health. J Sch Health 2010; 80(2):96-103.

9. Pelegrini A, Silva DAS, Petroski EL, Gaya AC. Sobrepeso e obesidade em escolares brasileiros de sete a nove anos: dados do projeto Esporte Brasil. Rev Paul Pediatr. 2010; 28(3):290-295.

10. Instituto Brasileiro de Geografia e Estatística. Pesquisa Nacional de Saúde do Escolar, 2012. Rio de Janeiro: IBGE; 2013. 256 p.

11. World Health Organization. Global strategy on diet, physical activity and health. Geneva: WHO; 2004.

12. James J, Thomas P, Cavan D, Kerr D. Preventing childhood obesity by reducing consumption of carbonated drinks: cluster randomised controlled trial. BMJ 2004; 328(7450):1237.

13. Fernandes PS, Bernardo CO, Campos RMMB, Vasconcelos FAG. Avaliação do efeito da educação nutricional na prevalência de sobrepeso/obesidade e no consumo alimentar de escolares do ensino fundamental. J Pediatr. 2009; 85(4):315-321.

14. Friedrich RR, Schuch I, Wagner MB. Efeito de intervenções sobre o índice de massa corporal em escolares. Rev Saúde Pública 2012; 46(3):551-560.

15. Brasil. Fundo Nacional de Desenvolvimento da Educação. Programa Nacional de Alimentação Escolar (PNAE) [Internet]. Brasília: MEC. [acesso em 03 maio 2015]. Disponível em: http://www. fnde.gov.br/programas/alimentacao-escolar/alimentacao-escolar-apresentacao

16. Lobstein T, Baur L, Uauy R. Obesity in children and young people: a crisis in public health. Obes Rev. 2004; 5(Suppl 1): 4-104. 
17. Brasil. Ministério da Saúde. Secretaria de Atenção à Saúde, Departamento de Atenção Básica. Experiências estaduais e municipais de regulamentação da comercialização de alimentos em escolas no Brasil: identificação e sistematização do processo de construção e dispositivos legais adotados. Brasília: Ministério da Saúde; 2007.

18. Minas Gerais. Lei no 18.372. Acrescenta dispositivos à lei no 15.072 , de 5 de abril de 2004, que dispõe sobre a promoção da educação alimentar e nutricional nas escolas públicas e privadas do sistema estadual de ensino. 2009 set 4. Diário do Executivo 05 set. 2009.

19. Gabriel CG, Ricardo GD, Vasconcelos FAG, Corso ACT, Assis MAA, Di Pietro PF. Alimentos comercializados nas escolas e estratégias de intervenção para promover a alimentação escolar saudável: revisão sistemática. Rev Inst Adolfo Lutz 2011; 70(4): 572-583.

20. Domene SMA. A escola como ambiente de promoção da saúde e educação nutricional. Psicol USP 2008; 19(4):505-517.

21. Leite FHM, Oliveira MA, Cremm EC, Abreu DSC, Maron LR, Martins PA. Oferta de alimentos processados no entorno de escolas públicas em área urbana. J Pediatr. 2012; 88(4):328-334.

22. Minas Gerais. Resolução no 1511. Orienta a aplicação da Lei 18.372/2009 no âmbito das escolas do sistema estadual de ensino. 2010 fev 26. Diário do Executivo, 27 fev. 2010.

23. Minas Gerais. Secretaria Estadual de Saúde. Subsecretaria de Vigilância e Proteção à Saúde. Superintendência de Vigilância Sanitária. Guia de ações de vigilância sanitária: 2013. Belo Horizonte: Secretaria Estadual de Saúde; 2013.

Recebido: $17 / 11 / 2015$

Revisado: 15/3/2016

Aceito: 15/6/2016 